\title{
Performance of a double metal n-on-n and a Czochralski silicon strip detector read out at $40 \mathrm{MHz}$
}

\author{
J.P. Palacios, ${ }^{\text {a }}$ A. Bates, ${ }^{\mathrm{b}}$ J. Buytaert,${ }^{\mathrm{c}}$ P. Collins, ${ }^{\mathrm{c}}$ \\ D. Eckstein, ${ }^{c}$ J. Harkonen, ${ }^{d}$ E. Tuovinen ${ }^{d}$ P. Luukka ${ }^{d}$

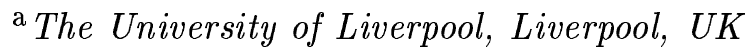 \\ ${ }^{\mathrm{b}}$ The University of Glasgow, Glasgow, UK \\ ${ }^{\mathrm{c}}$ CERN, Geneva, Switzerland \\ ${ }^{\mathrm{d}}$ Helsinki Institute of Physics, Helsinki, Finland
}

\begin{abstract}
The R\&D undertaken by the VELO group in order to produce a sensor that satisfies the tight radiation hardness, efficiency, resolution and low material requirements of LHCb has resulted in the choice of an n-on-n double metal layer solution. First measurements of the performance of the latest prototype and its related front end electronics, designed to function at the LHC speed of $40 \mathrm{MHz}$, are presented here. In addition, research has been carried out into new materials which could retain good performance in high radiation environments at and beyond the LHC, and could provide an alternative for a possible VELO upgrade. For the first time a full size Czochralski silicon detector sample with $50 \mu \mathrm{m}$ pitch strips has been irradiated with high energy protons and its performance has been measured in a test beam with 40 $\mathrm{MHz}$ electronics. The results of this test will be presented.
\end{abstract}

\section{Introduction}

LHCb is a dedicated B-hadron physics experiment at the Large Hadron Collider (LHC). It is a single arm small angle forward spectrometer designed to detect and reconstruct events in which $\mathrm{B}$ mesons are produced. The VErtex LOcator (VELO) acts as vertex detector and tracker for $\mathrm{LHCb}$ and is used in the second and third level triggers (L1 and L2 respectively in $\mathrm{LHCb}$ nomenclature) [1]. The VELO is particularly exposed to the harsh radiation environment present at the LHC due to its close proximity to the interaction point, with the closest strips receiving around $1 \times 10^{14} \mathrm{n}_{\text {eq }}$ per year. For this reason the VELO group has pursued an extensive R\&D program in order to 
find the best technology to meet the tight requirements of the VELO and LHCb. This has resulted in the choice of thin n-on-n silicon strip $\mathrm{R}$ and $\phi$ measuring sensors with double-metal for the first generation VELO. This solution is expected to be operational for two to three years of LHC running. An upgrade after this period presents an opportunity to employ new sensor technologies in order to improve the performance and radiation hardness of the detector. The VELO group is involved in research into new technologies, for example Czochralski, p-bulk float zone silicon (FZ-Si) [2], and 3D detectors [3]. This manuscript presents first test beam results of a thin n-on-n $R$ measuring sensor coupled to a Beetle1.2 readout chip and read out at $40 \mathrm{MHz}$. This sensor and chip combination is close to the final design. We also present the first test beam results of an large scale Czochralski p-on-n strip sensor after inhomogeneous irradiation.

\section{Baseline sensor and readout chip solution}

In order to satisfy the requirements mentioned above, the VELO group has opted for n-on-n, oxygenated, thin, fine pitch $\mathrm{R}$ and $\phi$ measuring sensors. Figure 1 shows a schematic of the strip and routing line layout for both sensors. $\mathrm{N}$-on-n silicon was chosen so that, after type inversion due to irradiation, the depletion zone evolves from the strip-bulk junction. This makes it possible to run parts of the detector below full depletion voltage without suffering from losses in resolution and efficiency $[1,4]$. The so-called PR03 is the final prototype sensor, differing only in small details from the production version, PR04, which will be available in May 2004. It was used for the tests described in this paper. The Beetle is the chosen readout chip for the VELO [5]. It will operate in $40 \mathrm{MHz} 4$-output analogue mode, with each output reading 32 channels. It is highly configurable and its pulse shape response can be carefully tuned to optimise the signal to noise ratio $(\mathrm{S} / \mathrm{N})$ and the signal remainder 25 ns after the peak time, known as the overspill.

\section{$3 \quad \mathrm{R}$ measuring prototype test beam}

An $200 \mu \mathrm{m}$ thick R measuring PR03 sensor coupled to a Beetle1.2 chip reading out 128 channels in LHC mode was tested in the X7 $120 \mathrm{GeV}$ pion beam at CERN in the Autumn of 2003. The mean pitch of the region read out was $44 \mu \mathrm{m}$. This was the first beam test of a thin n-on-n double metal sensor at LHC speeds. The aim of the test was to check the fulfillment of the VELO requirements with a sensor and readout chip close to the final design. Quanti-

ties such as signal, $\mathrm{S} / \mathrm{N}$, and overspill were evaluated for a different values of 
the Beetle shaper feedback voltage, $\mathrm{V}_{f s}$, which was found to be the most relevant parameter for these quantities [6]. In order to investigate the whole pulse shape, including the low signal and undershoot tail, tracks from the VELO test beam telescope were extrapolated to the test sensor and the charge in the four strips surrounding the interception point was summed. The $\mathrm{S} / \mathrm{N}$ is defined as this sum, divided by the average noise of the four strips [7]. The integrated $\mathrm{ADC}$ and $\mathrm{S} / \mathrm{N}$ distributions were plotted as a function of TDC. A Landau convoluted with a Gaussian was fitted to the ADC and S/N distribution of each TDC bin in order to obtain the most probable value for the peak (MPV).

\section{$4 \quad \mathrm{R}$ measuring prototype results and conclusions}

The MPVs for the signal amplitude and S/N as a function of TDC can be seen in Figures 2 and 3. The $\mathrm{S} / \mathrm{N}$ and overspill values, together with the values expected for a $300 \mu \mathrm{m}$ thick sensor, are shown as a function of $\mathrm{V}_{f s}$ in Figures 4 and 5. It is clear that the $\mathrm{LHCb}$ requirements of $\mathrm{S} / \mathrm{N}$ and overspill are not met by this sensor and readout chip combination. Therefore it is advisable to use $300 \mu \mathrm{m}$ thick silicon for the $\mathrm{R}$ measuring sensors of the first VELO detector.

\section{Future technologies: Czochralski silicon}

Czochralski silicon ( $\mathrm{Cz}-\mathrm{Si})$ is the standard, cheaper method of producing mono-crystalline silicon and is used by most IC manufacturers. It typically results in silicon that is of too low a resistivity to be used in HEP applications. However, a beneficial aspect of $\mathrm{Cz}-\mathrm{Si}$ is its high oxygen content, which can be three orders of magnitude higher than standard FZ-Si and one higher than diffusion oxygenated FZ-Si. This should make it very radiation hard. Furthermore, there is some evidence that its depletion behaviour is more stable against irradiation [8]. Recently it has become possible to manufacture higher resistivity Cz-Si, and a full scale strip sensor was fabricated in 2002. The sensor is $380 \mu \mathrm{m}$ thick, p-on-n with $50 \mu \mathrm{m}$ pitch and strip length $6.2 \mathrm{~cm}$. Its resistivity was measured as $1150 \Omega \mathrm{cm}$ and its depletion voltage as $420 \mathrm{~V}$ [9].

\section{$6 \quad$ Cz-Si test beam}

In order to see if such a sensor could be irradiated and read out at LHC speeds, the sensor was bonded to three SCTA readout chips and placed in the VELO 
$\mathrm{X} 7$ test beam setup before and after being irradiated to $7.8 \times 10^{14}$ protons $/ \mathrm{cm}^{2}$. The irradiation was non-uniform, and data were split into irradiation levels corresponding to $0.5,2$ and 3 years of $\mathrm{LHCb}$ running. The signal level was estimated using tracks in the same way as in the analysis described above. Signal values were obtained for the three irradiation levels and various bias voltages in order to see the charge collection characteristics of the sensor as function of fluence.

\section{$7 \quad \mathrm{Cz}-\mathrm{Si}$ results and conclusions}

The signal MPVs in ADC counts as a function of bias voltage for the three irradiation zones can be seen in Figure 6. These results are preliminary. The effect of irradiation on charge collection efficiency can be seen clearly. For this data taking the bias voltage was not raised as high as the nominal depletion voltage for the non-irradiated sensor, which was 420 volts. However it can be seen that at 371 volts the charge collection efficiency at $4.25 \times 10^{14} \mathrm{p} / \mathrm{cm}^{2}$ is more than $80 \%$ that at the lower irradiation level of $1.25 \times 10^{14} \mathrm{p} / \mathrm{cm}^{2}$, and after a very large dose of $7 \times 10^{14} \mathrm{p} / \mathrm{cm}^{2}$ it has dropped to around $50 \%$, which is still a good performance for such a heavily irradiated, thick sensor. Higher voltage data are not available for the irradiated sample, but an analysis of non-irradiated data is underway in order to determine the absolute full charge collection value and depletion point of the sensor. This is the first time that such a large scale sensor has been built and operated under such conditions, and the results look reasonably promising for future investigations.

\section{References}

[1] LHCb Vertex Locator Technical Design Report, CERN/LHCC 2001-011.

[2] G. Casse, Nucl. Instr. and Meth. A (2004), these Proceedings.

[3] RD50 Status Report 2002/2003, CERN-LHCC-2003-058, 14.11.2003.

[4] J. Libby, Evaluation of fine pitch irradiated silicon microstrip detectors for LHCb, JHEP (2001) PRHEP-hep2001/263.

[5] N. Van Bakel et al. The Beetle Reference Manual, LHCb 2001-46 ELECTRONICS (2001).

[6] J. Buytaert et al., First measurement of the performance of a Beetle1.2 chip reading out a VELO sensor, LHCb-2003-133 VELO.

[7] J.P. Palacios, Performance of a Beetle1.2 chip reading out a Micron PR03 R measuring sensor, LHCb 2003-135 VELO (2004). 
[8] G. Lindstroem, Recent results from the HH-CiS Collaboration, First RD50 Workshop, CERN, 02/04 October 022004.

[9] J. Harkonen et al. Nucl. Instr. and Meth. A 514 (2003) 111.

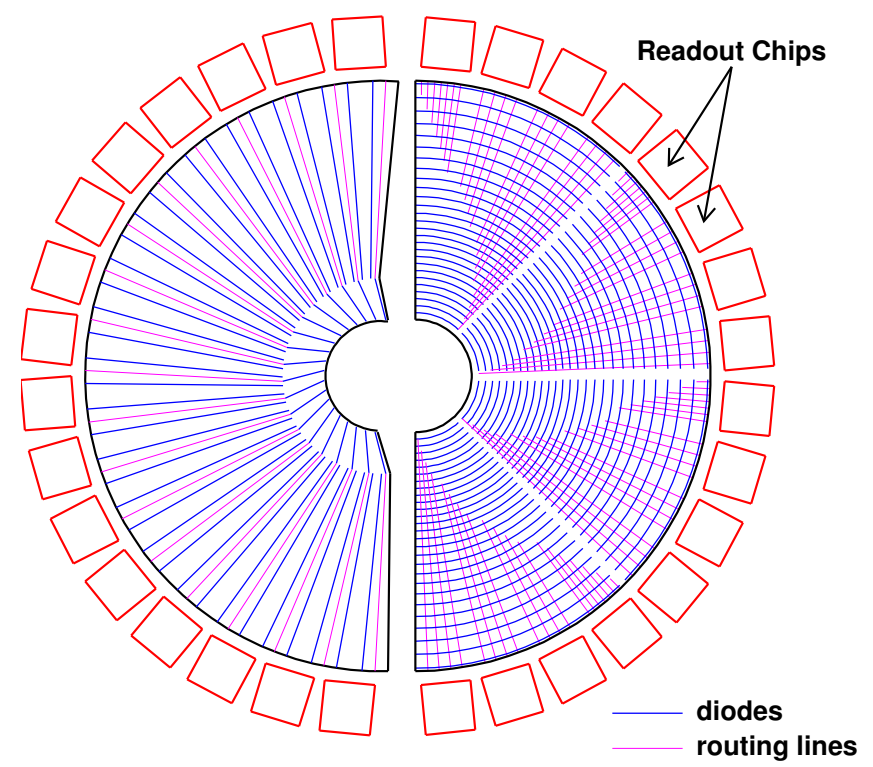

Fig. 1. A schematic of the strip and routing line layout for the production prototype VELO sensors, PR04. 


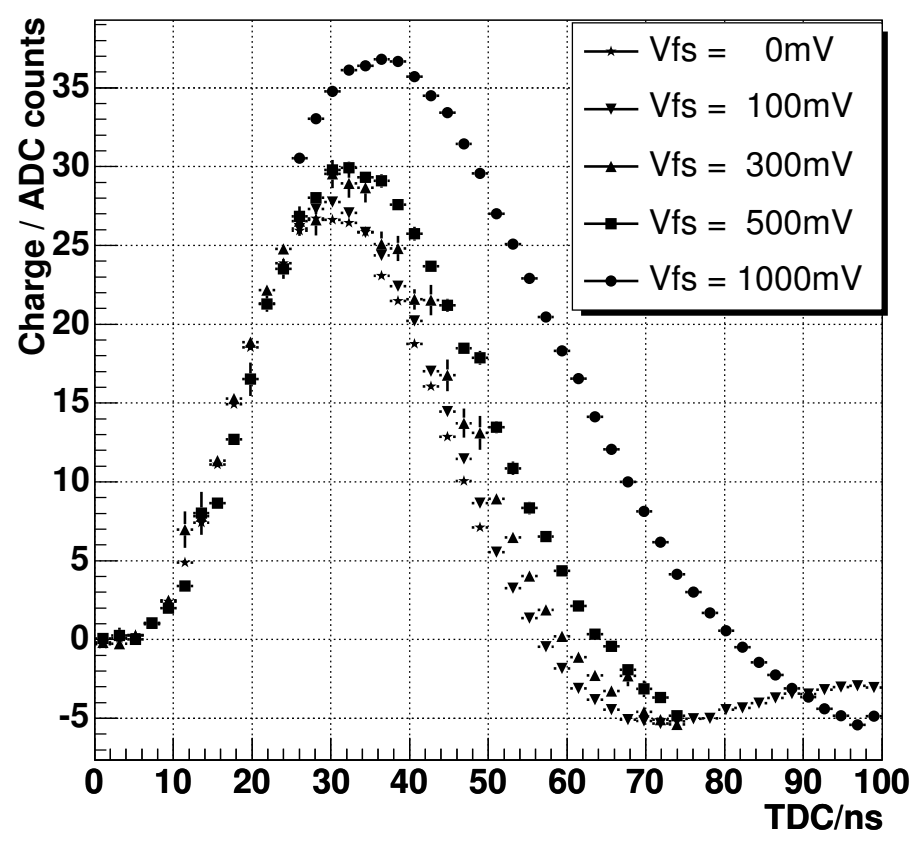

Fig. 2. MPVs, in ADC counts, from Landau-Gaussian fits to integrated charge distributions as a function of calibrated TDC (ns) for different Beetle $V_{f s}$ settings.

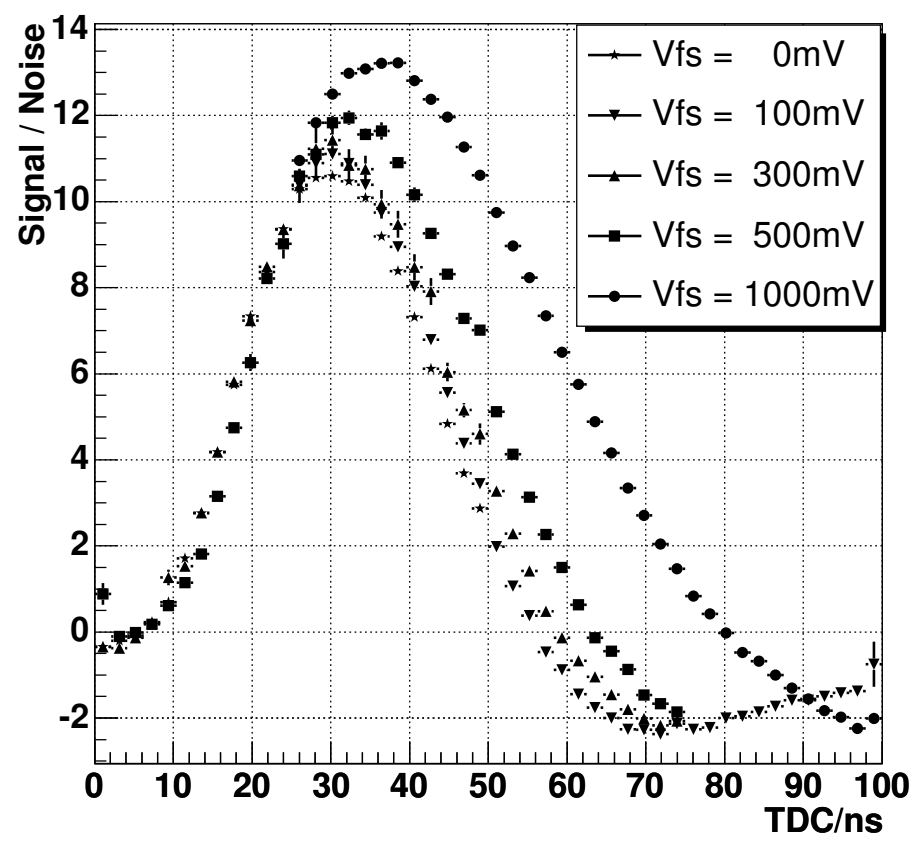

Fig. 3. MPVs from Landau-Gaussian fits to $\mathrm{S} / \mathrm{N}$ distributions as a function of calibrated TDC (ns) for different Beetle $V_{f s}$ settings. 


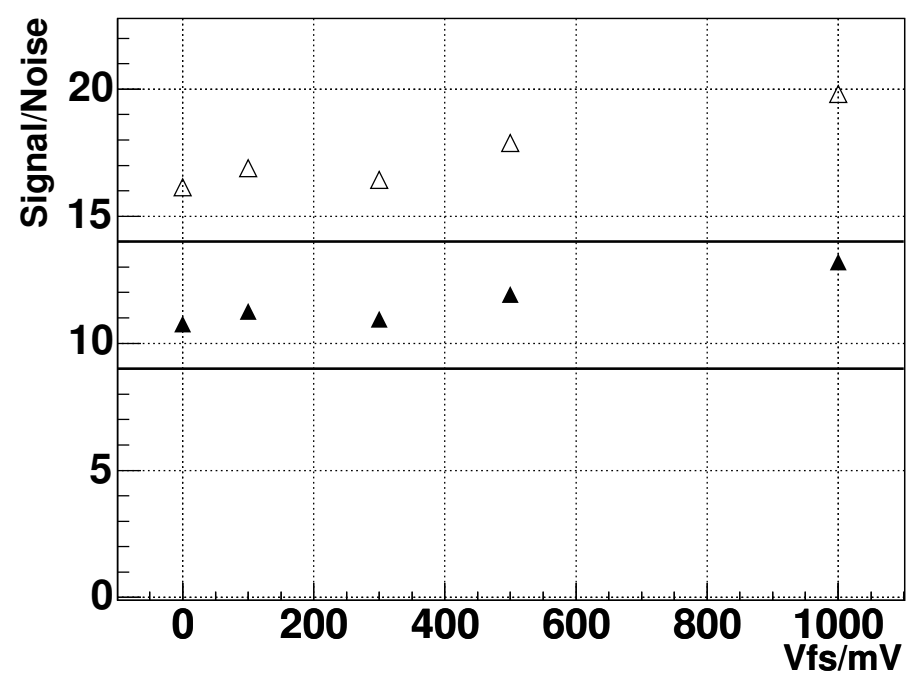

Fig. 4. $\mathrm{S} / \mathrm{N}$ values as a function of $\mathrm{V}_{f s}$ for the data (black triangles) and extrapolation to $300 \mu \mathrm{m}$ sensor (open triangles). The horizontal lines mark the LHCb starting requirement of 14 , and the trigger minimum requirement of 9 .

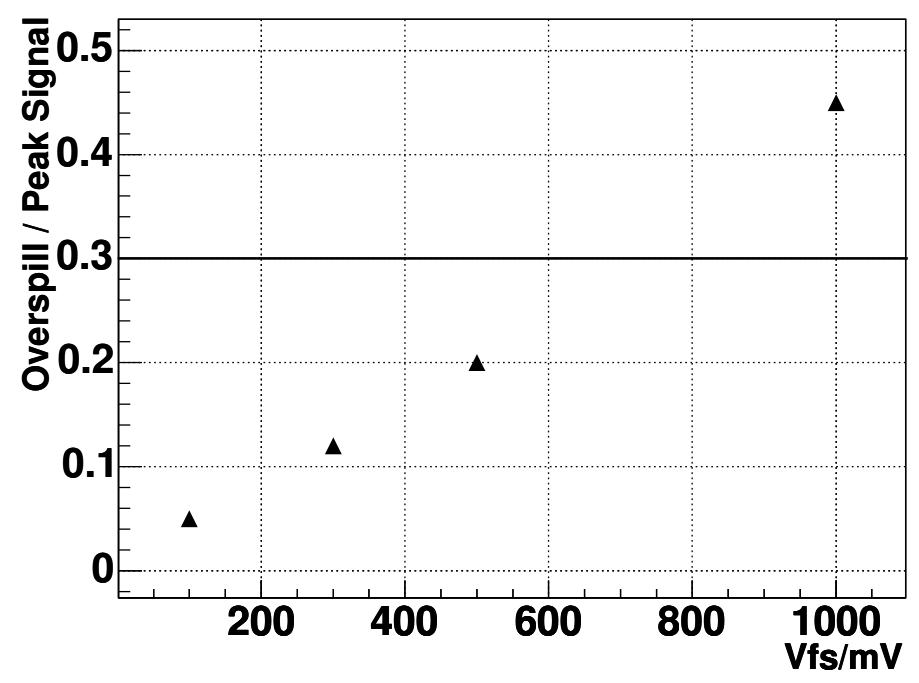

Fig. 5. Ratio of overspill to peak signal as a function of $\mathrm{V}_{f s}$. 


\section{Most Probable Signal as a function of Bias Voltage}

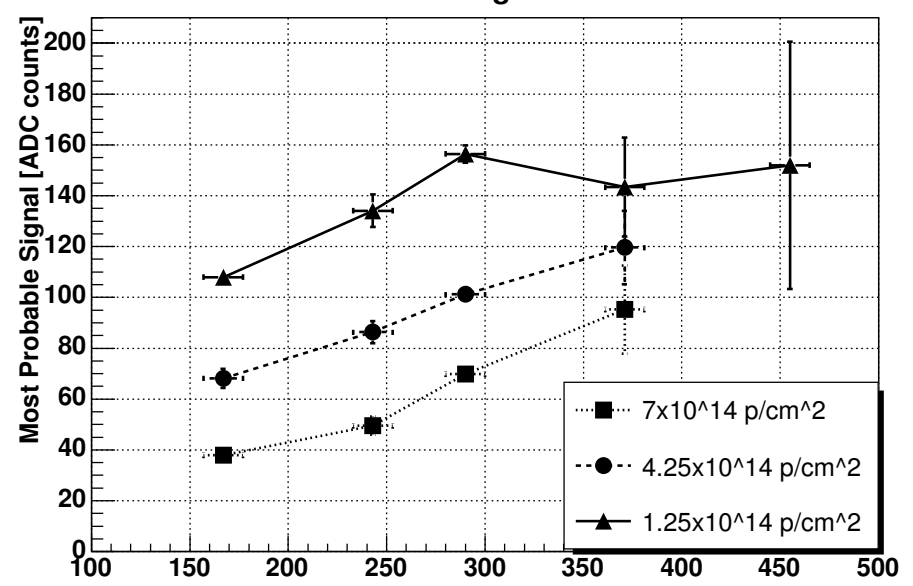

Fig. 6. Signal MPVs in ADC counts as a function of bias voltage for the three irradiation zones on the full size Czochralski silicon prototype. 